\title{
4G Terminal LTE-CPE in the Practical Application
}

Xianjing Zhang ${ }^{1, a}$ and Hanbing $\mathrm{Chu}^{1, b}$

${ }^{1}$ Information Engineering Institute, Zhengzhou University of Industrial Technology, Xinzheng, 451100, China

a1072746357@qq.com, $5554328207 @ q q . c o m$

Xianjing Zhang(1990- ), female, Native place of Henan, Zhoukou, the Title is Lecturer, Main Research Direction: Wireless Video Transmission

1072746357@qq.com.

\section{Keywords: LTE; 4G terminal; CPE; Modern wireless}

\begin{abstract}
LTE is the largest new technology research and development project initiated by 3GPP in recent years. With the improvement and maturity of LTE technology standards and the operators on the LTE investment increasing, 4G terminal products have appeared and the scope of its application is more and more widely. 4G terminal products LTE-CPE has been used in a variety of scenarios: airport, train station, campus, bus, subway, small and medium enterprises, restaurants, cafes, individual businesses, residential users and so on. This paper introduces $4 \mathrm{G}$ terminal products CPE in the practical application.
\end{abstract}

\section{Introduction}

Two of the most important bases in modern wireless communication systems are Multiple Access and Multiplexing. Multi-access technology provides the possibility for different users to access the wireless communication network simultaneously, and Multiplexing technology provides the possibility for users to receive and send data simultaneously. [1]The development process of wireless communication from $2 \mathrm{G}, 3 \mathrm{G}$ and $3.9 \mathrm{G}$ to $4 \mathrm{G}$ is basically continuous improvement in these two technologies. The development and evolution of wireless communication technology is shown in fig. 1.

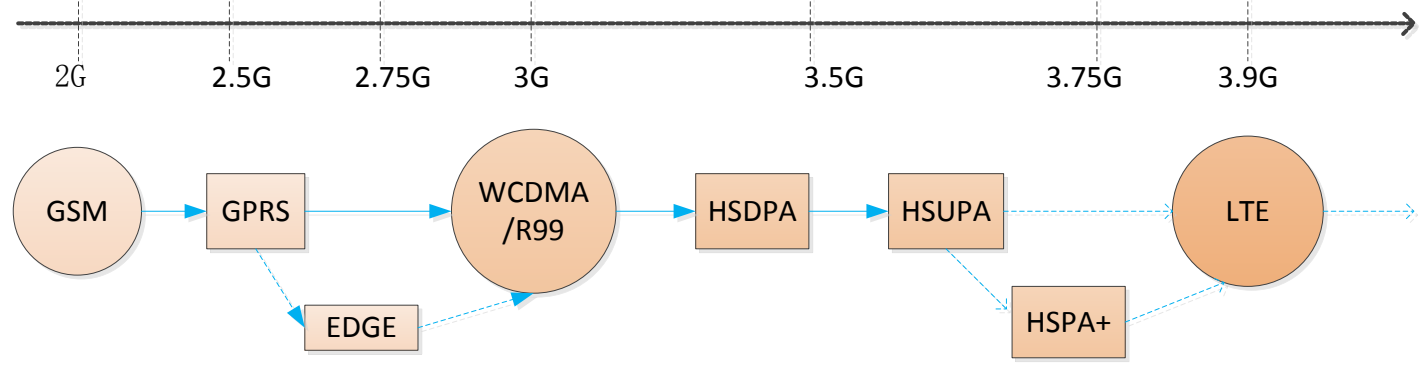

Figure 1. The development and evolution of wireless communication technology

3GPP started LTE related standard work in December 2004, and LTE is a project on UTRA and UTRA improvement. The research work of LTE is divided into two phases according to the work flow of 3GPP: SI (Study Item, technical feasibility study stage) and WI (Work Item, technical specification writing stage)[2].

$4 \mathrm{G}$ is the fourth generation of mobile communication technology. The $4 \mathrm{G}$ standard originally adopted by China mobile is TD-LTE, which is one of the two most common 4G standards in China. $4 \mathrm{G}$ terminal product CPE has been used in many scenarios. In the light of the development of wireless communication, CPE will become more and more widely used. CPE is a device for converting high-speed $4 \mathrm{G}$ signals into $\mathrm{WiFi}$ signals, and its $\mathrm{AP}$ function and router performance are stronger. CPE can be used simultaneously by various mobile terminals such as tablet PC, smart 
phone and notebook, with a complete user authentication function and billing function.[3]

\section{Application of CPE}

In order to realize the full coverage of LTE network, TD-LTE CPE products which are suitable for a variety of scenes should be born. LTE-CPE includes indoor type, outdoor type, vehicle type, etc.. The outdoor type CPE, which adopts the all-enclosed waterproof design, ADAPTS to the outdoor working scene under the harsh conditions. Vehicle-mounted CPE, designed for vehicle-mounted communication, has the advantages of good anti-shock effect and strong power supply.

LTE - CPE is a system fusion product, which USES the mobile communication system 3G/LTE to provide the return transmission channel, while the $\mathrm{WiFi}$ implementation is covered. In the early days of LTE construction, the LTE - CPE, which is covered by WiFi, can be used to solve the problem of the low end of TD-LTE, and the $2 \mathrm{G} / 3 \mathrm{G}$ phone terminal is not compatible with LTE. In the application scenario, LTE-CPE is flexible and can meet the requirement of WiFi coverage for many special occasions. [4] LTE-CPE is mainly used in mobile scene and fixed scene with difficulty in wired transmission construction. LTE-CPE can compensate for the limitations of wired transmission, and its flexible mesh mode can adapt to different wireless or mobile scene requirements, as shown in fig. 2.

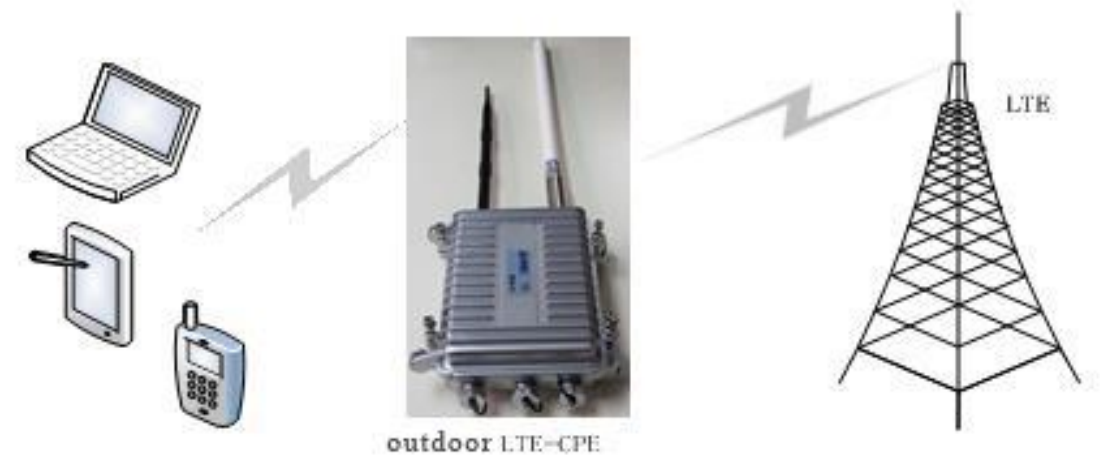

Figure 2. The application of CPE

The specific application scenarios are as follows:

(1) the family members of the community -- in the situation where there is no wireless broadband access, users need to solve the need to surf the Internet at anytime and anywhere in the room.

(2) small and medium-sized enterprises, hotels, cafes, which has many features such as user, liquidity, large business demand, and more terminal form. It needs to solve the need of wifi access, Internet access, fixed-line, fax and so on. [5,6]

(3) campus, which has many features such as a large number of users and large data business needs, mainly including web browsing, information query, online games, movies, music, video, QQ, etc.

(4) Buses and subways, which has many features such as the number of users, the large amount of liquidity, the Internet demand, mainly to browse the web, e-books, games, video.

(5) Railway station, city business district, bustling street, shopping mall, airport, square, which has many features such as population density, strong liquidity, large demand for data business, different terminal form.[7]

(6) in the Internet of things and other fields, mainly including high-definition video surveillance.

Standard WiFi client uplink power is limited(generally $15 \mathrm{dBm})$, regardless of the downlink power of the wireless coverage base station, the uplink distance of the standard WiFi client is limited by uplink power, so the distance is very limited. Wireless outdoor CPE distance can reach the standard WiFi client several times.

Wireless CPE is a wireless terminal access device that receives wifi signal, which can replace 
wireless network card and other wireless client devices. Wireless routers, wireless AP, wireless base stations and other wireless signals can be received, which is a new type of wireless terminal access device. CPE can be widely used in rural, urban, hospital, unit, factory, residential area and other wireless network access, which can save the cost of laying cable network.[8]

\section{The Application of CPE in the Countryside}

With the continuous development of rural economy in China, rural digital construction is on the agenda. The development and application of Internet and modern information technology means to create favorable conditions for the development and sustainable development of agriculture and rural areas. At present, the "digital agriculture construction", "agricultural information service network", "the Internet project of ten thousand households" and "remote education of modern farmers" are flourishing in rural areas of China.

Rural broadband access includes traditional wired access mode and wireless broadband access mode.

(1) Traditional wired access mode. The carrier's ADSL or optical fiber access is often restricted by rural geographical conditions. ADSL has requirements for transmission distance, and its introduction line is only about three kilometers. $[9,10]$

(2) Wireless broadband access mode. Wireless broadband has many advantages such as advanced technology, high bandwidth, stable and flexible development. It is the first choice for rural network construction.

The current challenges for rural wireless broadband include base stations and clients.

(1) Base stations. The launch power of AP is limited, so that the signal cannot be covered far enough. So if you want a wide coverage, you need to add a sufficient number of AP to the coverage. In the township with much land and few people by increasing the AP number to expand coverage, it can be said that the way of full coverage is not worth the loss.

(2) Clients. Even if the base station has enough emission power to cover a few tens of kilometers, but because of the two-way nature of data transmission, the power of the PC wireless card is usually only $50 \mathrm{~mW}-60 \mathrm{~mW}$, and the antenna is generally hidden in the body. It has no good direction. So a STA can receive a good signal from AP, but the AP doesn't get a STA signal.

In response to the above problems, we adopt the "wireless network (WLAN) coverage + outdoor CPE mode" to introduce the broadband network. So that it can make Internet information into every household in the village, and let every modern villager use the Internet to disseminate information. An outdoor wireless base station is designed in the vicinity of several natural village central and covers the main area of the village through directional antennas. The outdoor CPE reception device is installed outside the village to enhance the reception effect of the wireless network signal. The outdoor CPE access system is very extensible and can realize the transmission of multiple data services. Such as video, security monitoring, voice, data exchange and so on, users can get high bandwidth multimedia enjoyment, as shown in fig. 3 .

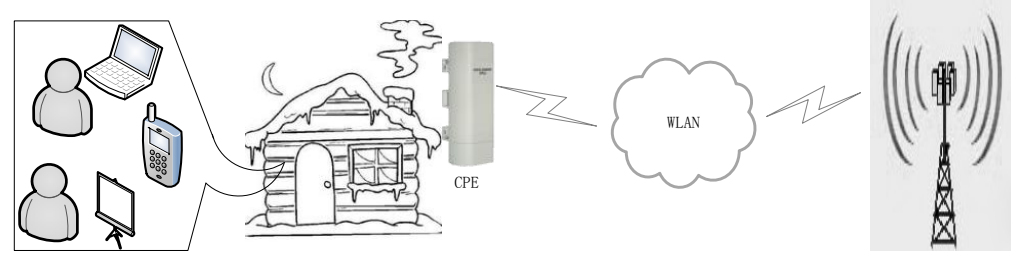

Figure 3. The application of CPE in the countryside

The WiFi wireless outdoor CPE, which conforms to the IEEE802.11b/g/n standard, has high transmission rate and high sensitivity to receive. It can establish a comprehensive coverage system of WLAN through the cooperation with WiFi base station equipment.

The main advantages and characteristics of this wireless broadband access scheme are as follows: 
(1) The outdoor CPE access method can quickly network, so that the system can be put into operation rapidly and shorten the investment recovery time, which can effectively reduce the implementation cost and maintenance cost of the whole coverage area network.

(2) In the implementation of wireless coverage and outdoor CPE access, taking full advantage of the existing network resources of operators, we can effectively reduce the cost of wireless systems.

(3) Short installation period, convenient maintenance and capacity expansion. The network is flexible and scalable, that is, it is ready to be installed and ready to be used. It is not necessary to lay the fiber lines for the newly added coverage area, so as to make it easy and fast to realize the dream of the rural users to surf the Internet.

\section{The Application of LTE-CPE in the City}

With the rapid development of the mobile Internet, more and more people use their mobile phones to surf the Internet to pass the hours of transportation. With the expansion of 4G LTE pilot cities and scope, people have access to free WiFi on buses. LTE - CPE transforms LTE signal into $\mathrm{WiFi}$ signal, which can be used by smartphones, tablets and laptops etc.. The WiFi signal is easy to access and the bandwidth is very high. It can make people easy to surf the Internet, and technology brings us a convenient life experience. The application plan is shown in fig. 4.

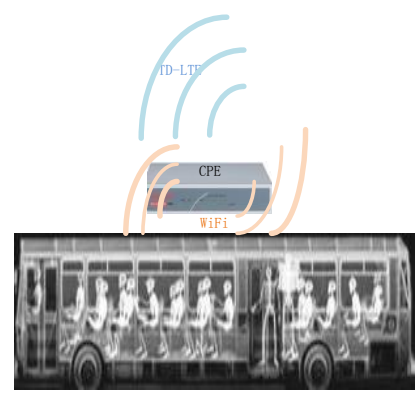

Figure 4. The Application of LTE-CPE in the City

LTE-CPE not only can be applied to the field of public transport industry, but also can be used in smart city, digital city management, emergency system, safe city and other information construction. It can also be applied to the construction of wireless broadband in many industry fields.

\section{Looking into the Future}

With the expansion of 4G network coverage, as well as 4G terminal products (support TD-LTE 4G network mobile phones, data cards, CPE, etc.) have come to market, $4 \mathrm{G}$ network has ushered in its golden age. With the popularization of mobile terminal, more and more people are entering the age of mobile life and enjoying the convenience and efficiency brought by mobile Internet. At the same time, China's $4 \mathrm{G}$ base stations are still in a rapid construction cycle, with $2 / 3 \mathrm{G}$ users accelerating to 4G migration, and $4 \mathrm{G}$ users continue to shoot up. The development of $4 \mathrm{G}$ industry chain in China has been one of the highest in the world, and the development of $4 \mathrm{G}$ will also promote the development of the whole Internet.

\section{References}

[1] F.D. Liu. The Hardware Research and Design of the Integrated Terminal of the on-board HD Video Monitoring System Based on Beidou and 4G Network Technology(MS., Shandong University, China 2016),p.35.

[2] T.C. Chen, Z. Wang, and K.H. Chen. Information Communication, Vol. 26(2016) No.2, p.261.

[3] Y. Zhou. Research on the Marketing Strategy of 4G Mobile 4G Terminal Based on Big Data(MS., Jiangxi University of Finance and Economics, China 2016),p.22. 
[4] Z.Y. Han. Research on the Management of 4G Terminal Promotion Project of Guangyuan Mobile (Ph.D., University of Electronic Science and Technology, China 2016),p.40.

[5] Y.T. Zhang. Forecasting and accurate marketing of 4G terminal telecom users in Shenzhen (MS., Fujian Normal University, China 2016),p.29.

[6] W. Lu. China Communication, Vol. 1(2014) No.1, p.122.

[7] S. Zheng and Q. Zhao. Telecommunications Technology, Vol. 29(2015) No.6, p.15.

[8] Q. Wang. Terminal-to-Terminal Calling for GEO Broad band Mobile Satellite Communication (Ph.D., University of Electronic Science and Technology, China 2015),p.33.

[9] T. Wang and M.Z. Xu. China Telecom, Vol. 12(2015) No.4, p.42.

[10]G.S. Wang. Intelligent terminal information security in 4G era(MS., Zhengzhou University, China 2016),p.30. 